\title{
AN OPERATOR TRAINING FACILITY AT THE ADVANCED PHOTON SOURCE*
}

\author{
J.W. Lewellen ${ }^{\dagger}$, S. Pasky, ANL, Argonne, IL 60439, USA
}

\section{Abstract}

The Advanced Photon Source (APS), a premier thirdgeneration synchrotron radiation source, presently operates in top-up mode during most user run time. During top-up mode, charge is injected into the storage ring at approximately 2-minute intervals to stabilize the stored beam current to within $0.1 \%$. Top-up mode requires the dedicated use of the entire APS injector system, severely limiting time available for operator training.

The APS injector test stand (ITS) is presently configured to operate a ballistic bunch compression (BBC) rf gun, which uses three independently powered and phased rf cavities, and either a thermionic cathode or a photocathode. Operation of the $\mathrm{BBC}$ gun requires cavity phasing and input rf power selection analogous to the operation of a high-energy linac. In conjunction with the ITS beamline components (quadrupoles, dipoles, diagnostics, correctors), this provides an effective analog to the APS linac. Operators can therefore practice accelerator tuning and control methods with a live beam and real components, rather than simulations, while top-up operation is ongoing.

\section{INTRODUCTION}

For some time, the Advanced Photon Source has been operated primarily in top-up mode during user studies. During top-up, charge is injected into the storage ring once every two minutes, to maintain a stable circulating beam current. This eliminates the varying heat load on user optics associated with stored beam current decay and subsequent refills, providing for more stable operation and constant, maximum flux operation. Top-up has also allowed the APS to operate using lower emittance, higher average brilliance lattices, with associated shorter storedbeam lifetimes, to further improve user operations.

Top-up operation comes with a price, however, both in terms of machine maintenance requirements and in terms of injector availability for other uses. During top-up mode, the entire APS injector complex must essentially run continuously. Therefore, there are greater than usual demands upon operational reliability and performance. There is also very little time to fix injector faults before the stored beam current is impacted significantly.

This paper deals with the other measure of top-up costs: injector availability for non-storage-ring injection, specifically with regard to operator training time.

\footnotetext{
* This work is supported by the U.S. Department of Energy, Office of Basic Energy Sciences, under Contract No. W-31-109-ENG-38.

$\dagger$ Lewellen@aps.anl.gov
}

\section{APS TRAINING REQUIREMENTS}

Operators at the APS are required to be knowledgeable about four separate accelerators: the linac, the particle accumulator ring (PAR), the booster synchrotron, and the storage ring. (The PAR is slated for long-term obviation; however, it will remain in use for the next year at least.)

Operator training can be conducted only when the storage ring is not in top-up mode and the APS is not in a shutdown. Eight-hour and 2-day studies periods are alternately scheduled on a weekly basis, however, these are most often dedicated for machine repair and verification, and machine physics studies.

There are typically 2 to 3 weeks of non-top-up storage ring operations scheduled every 3 -month run period during which operator training time can reasonably be scheduled. This time, however, is in high demand also for injector maintenance, free-electron laser operation, and studies related to future operational requirements of the APS. Therefore, a means of providing operator training time during top-up operation is increasingly important.

\section{FUNDAMENTAL ELEMENTS}

An accelerator operator training facility should be able to recreate all of the fundamental aspects of generating, transporting, diagnosing, and using a particle beam.

For the APS, in many respects the most complex machine to operate correctly is the linac. It is the only noncirculating machine in the APS injector complex, and as a result, many of the controls and operating systems are somewhat different from the remainder of the APS accelerators. The linac is also the most in-demand accelerator for studies during non-top-up periods.

\section{LINAC SURROGATES}

A good linac simulator would certainly be a feasible means of providing operator training; however, there are several limitations to this approach. These include response time, construction time, and operator buy-in.

All of the physical features appearing in the APS linac have analogs in various simulation codes such as elegant [1] or PARMELA [2]. Even with a fast computer, the response time of these codes to changes in the input deck is not, generally speaking, as rapid as the response of a real accelerator to a similar change. There must also be a method for wrapping the simulation code, including inputs and outputs, into an analog of the actual control system, to foster both familiarity and the causal relationship with the actual linac control system.

Wrapping the required features into a graphical interface that mirrors the behavior and response of the actual APS control system, however, would take the dedicated 
attention of a number of programmers and physicists. While certainly feasible, this would consume scarce resources for what is essentially a single-purpose program. Finally, even a very good training program could elicit the "videogame effect." That is, the operators know it is not real; this can lead to a lack of interest, among other effects.

Instead, we propose that the APS injector test stand (ITS) can serve as a reasonable analog to the APS linac for training purposes. The ITS contains most of the elements found within the APS linac and all the essentials for learning the fundamentals of accelerator operation. Perhaps most importantly, the presently installed electron gun consists of three rf cavities, each with its own independent rf port. This allows independent adjustment of the gradient and phase in each cell, directly analogous to separate tanks or sectors in a high-energy linac.

Other components of the ITS line are common linac elements: quadrupoles, steering correctors, gate valves, dipoles, current monitors, Faraday cups, and viewscreens. A CAD-generated layout of the test stand is shown in Figure 1. In fact, the only element present in the APS linac that is missing an analog in the ITS is a beam position monitor (BPM). We plan to install a BPM in the ITS to permit the testing of new BPM electronics, however, so this will be addressed shortly.

The use of the ITS as an operator training facility has two additional advantages. First, operators may be shown the physical layout at any time, to permit an immediate comparison between the reality of the hardware versus its representation in the control system. Such an understanding can be valuable when attempting to remotely diagnose fault conditions. Second, if an operator is so inclined, he or she can participate in the active injector research program taking place in the test stand.

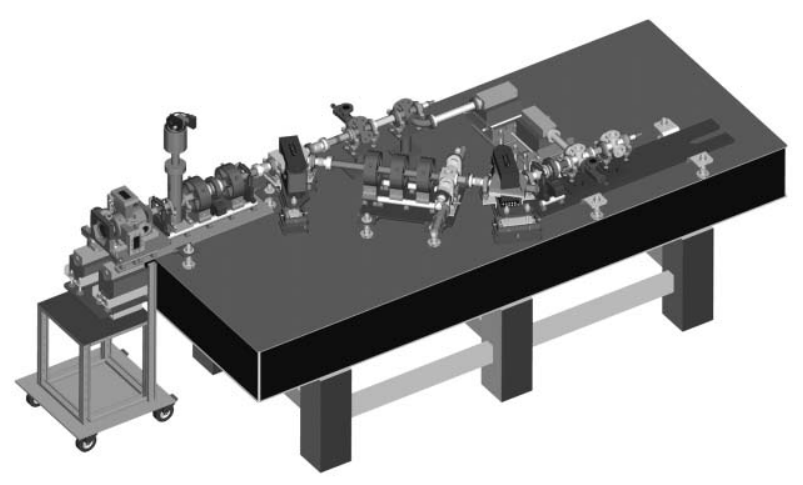

Figure 1: Injector test stand physical beamline layout.

\section{CONTROL SYSTEMS}

The ITS control system [3] is based on EPICS, as are all accelerators in the APS complex. This effectively leverages the wide variety of component control screens, hardware drivers, and higher-level software in use throughout the remainder of the APS accelerator complex. It also helps ensure that the "look and feel" and response of the ITS control system is similar to that of the rest of

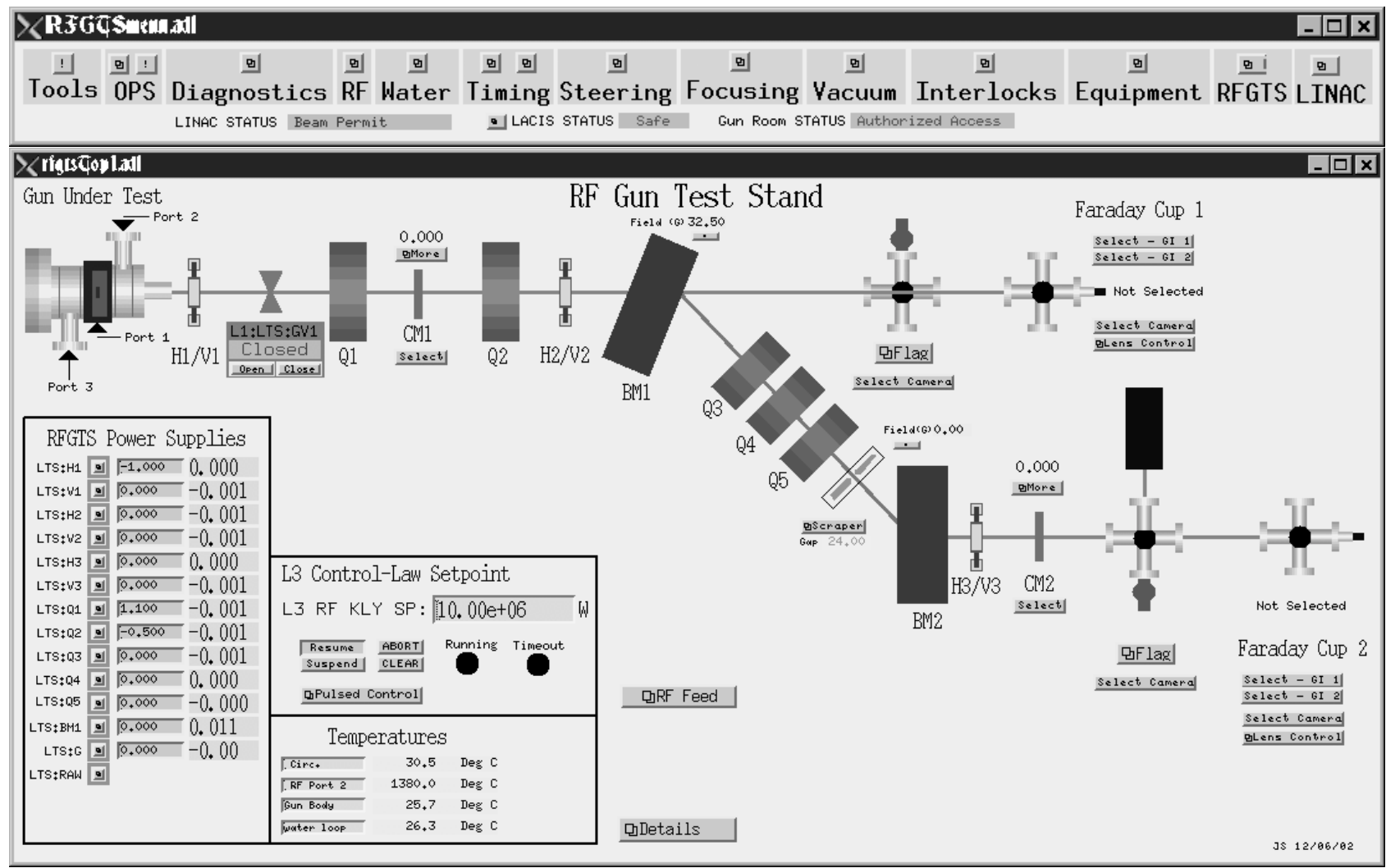

Figure 2: Injector test stand control and facilities screens. 
the APS. The ITS control system does not rely on APS operational hardware, however, meaning that faults or error conditions within the ITS will not affect the operation of the APS itself.

The top-level control screens for the ITS are shown in Figure 2. The layout of the beamline screen is chosen to mirror the actual device layout, as is done with the main APS accelerator. The facilities panel also mimics the layout and content of the APS linac.

The rf control system for the ITS [4] is, by necessity, different from that of the linac. Instead of individual klystrons for each linac sector, the ITS uses two variable power dividers, two phase shifters, and three circulators to provide isolated $\mathrm{rf}$ power feeds to three separate ports. The ITS rf power distribution control screen is shown in Figure 3.

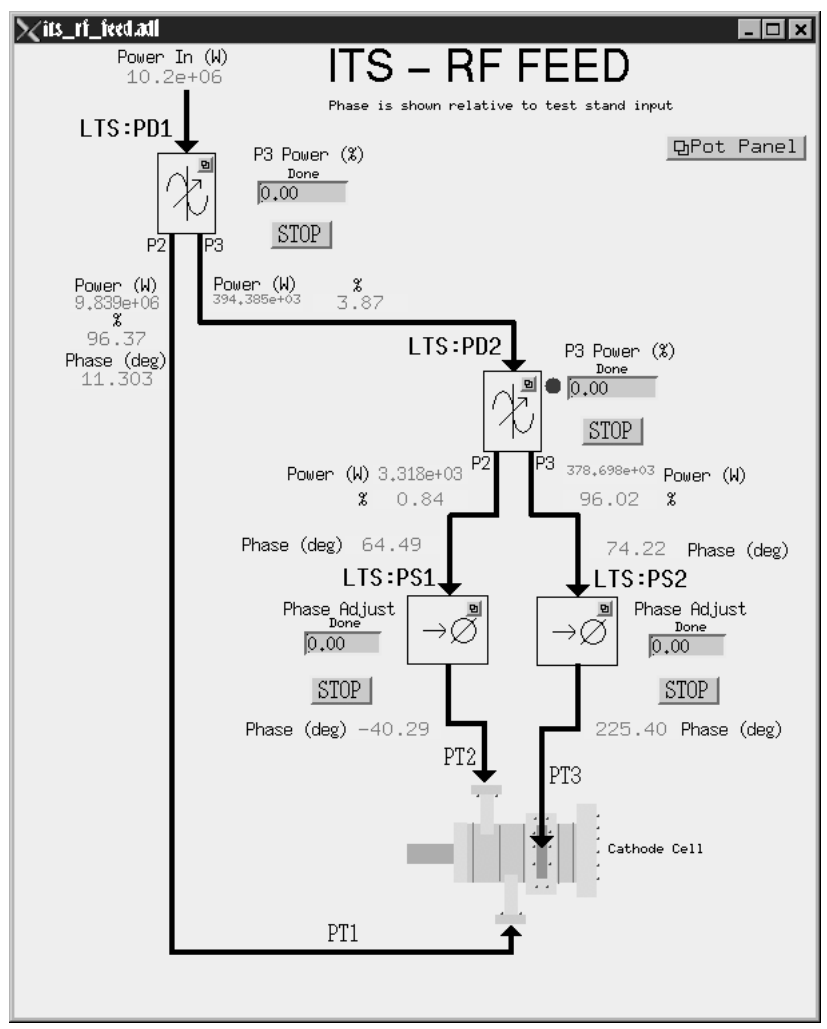

Figure 3: ITS rf power feed control screen.

Whenever possible, the individual component control panels, such as those for flags, power supplies and vacuum systems, use exactly the same template files as those for the APS linac.

\section{CONCLUSIONS}

The APS injector test stand is currently operational, and commissioning has begun on the 2.5 -cell ballistic compression rf gun. Although it is intended primarily for use as a research laboratory, the test stand will also serve as an operator training facility. Advantages include similarity of the control system to the APS as a whole, similarity of tuneup procedures to the APS linac, easy access for operator orientation and familiarization, and the ability to permit operators to actively participate in an ongoing research program.

\section{ACKNOWLEDGEMENTS}

Many people have contributed to the planning, construction, and operation of the Advanced Photon Source injector test stand; the authors in particular wish to thank Ned Arnold, Steven Berg, Debra Briddick, William Berg, Robert Dortwegt, George Goeppner, Aaron Lopez, John Maclean, and Wayne Michalek.

This work is dedicated to the memory of Kevin Beczek.

\section{REFERENCES}

[1] The elegant program (source and binaries) and manual can be obtained from the APS Operations Analysis Group website:

http://www.aps.anl.gov/asd/oag/oaghome.shtml

[2] J.H. Billen and L. Young, "PARMELA," Los Alamos document LA-UR-96-1835 (user's guide for version 3.30, revised 27 March 2003).

[3] J.F. Maclean and N.D. Arnold, "The Advanced Photon Source Injector Test Stand Control System," Proceedings of the $8^{\text {th }}$ International Conference on Accelerators and Large Experimental Physics Control Systems (ICALEPCS 2001), San Jose, CA, pp. 98100 (2001).

[4] A. Grelick et al., "The High Power S-Band Feed Subsystem for the Advanced Photon Source (APS) Injector Test Stand," Proceedings of the 2001 Particle Accelerator Conference, pp. 1393-1395 (2001). 